\title{
The homogeneneous balance method and its application to the Swift- Hohenberg equation
}

\author{
J. Biazar, M.barandkam \\ Department of Applied Mathematics, Faculty of Mathematical Sciences, University of Guilan \\ P.O. Box. 41635-19141, P.C. 41938336997, Rasht, Iran \\ E-mail addressesbiazar@guilan.ac.ir,matinbarandkam@yahoo.com.
}

\begin{abstract}
In this paper, homogeneous balance method (HBM) is proposed for seeking the traveling wave solutions for the SwiftHohenberg equation. In this approach HBM is applied to solve the Riccati equation and the reduced nonlinear evolution equations, respectively. Many new exact travelling wave solutions are successfully obtained. The method is straightforward and concise, and can be also applied to other nonlinear evolution equations.
\end{abstract}

Keywords: Use Homogeneous balance method; Traveling wave solutions; Soliton solutions; Riccati equation; Swift-Hohenberg equation.

\section{Introduction}

In recent years, Wang et al. presented a useful method called homogeneous balance method [1-3], for finding an exact solution of nonlinear partial differential equations. Fan and his collaborators improved the homogeneous balance method and made it more straightforward and simple [4-7]. However, they are restricted because the difficulty of finding solutions of equations, such as the Riccati equation. This conduct equations makes the homogeneous balance method used more extensively. This method can be used to find more exact traveling wave solutions of a given nonlinear partial differential equations.

In this paper, HB method is used to solve the Riccati equation $\phi^{\prime}=a \phi^{2}+b \phi+c$ and the reduced nonlinear ordinary differential equation for the Swift-Hohenberg equation, respectively.

For the the Swift-Hohenberg equation

$$
u_{t}+2 u_{x x}-\sigma u_{x x x}+u_{x x x x}=\alpha u+\beta u^{2}-\gamma u^{3} \text {. }
$$

Where $\sigma, \alpha, \beta$, and $\gamma$ are parameters of equation.

Let us consider the traveling wave solutions

$$
u(x, t)=u(\xi), \quad \xi=k x+l t+d,
$$

Where $k, l$ and $d$ are constants.

Substituting (2) into (1), results in

$$
l u^{\prime}+2 k^{2} u^{\prime \prime}-\sigma k^{3} u^{\prime \prime \prime}+k^{4} u^{(4)}=\alpha u+\beta u^{2}-\gamma u^{3} .
$$

We now seek the solution of Eq.(3) in the following form :

$$
u=\sum_{i=0}^{m} q_{i} \phi^{i} .
$$


Where $q_{i}$ are constants, to be determined, and $\phi$ satisfy the Riccati equation

$$
\phi^{\prime}=a \phi^{2}+b \phi+c
$$

Where $a, b$, and $c$ are constants. By balancing $u^{(4)}$ with $u^{3}$, one gets easily $m=2$. [4]

$$
u=q_{0}+q_{1} \phi+q_{2} \phi^{2}
$$

Substituting in (3), from, [5-6], collecting all terms with the same power $\phi^{i}(i=0,1,2,3,4,5)$ and setting each of the obtained coefficients to zero, yields the set of algebraic equations :

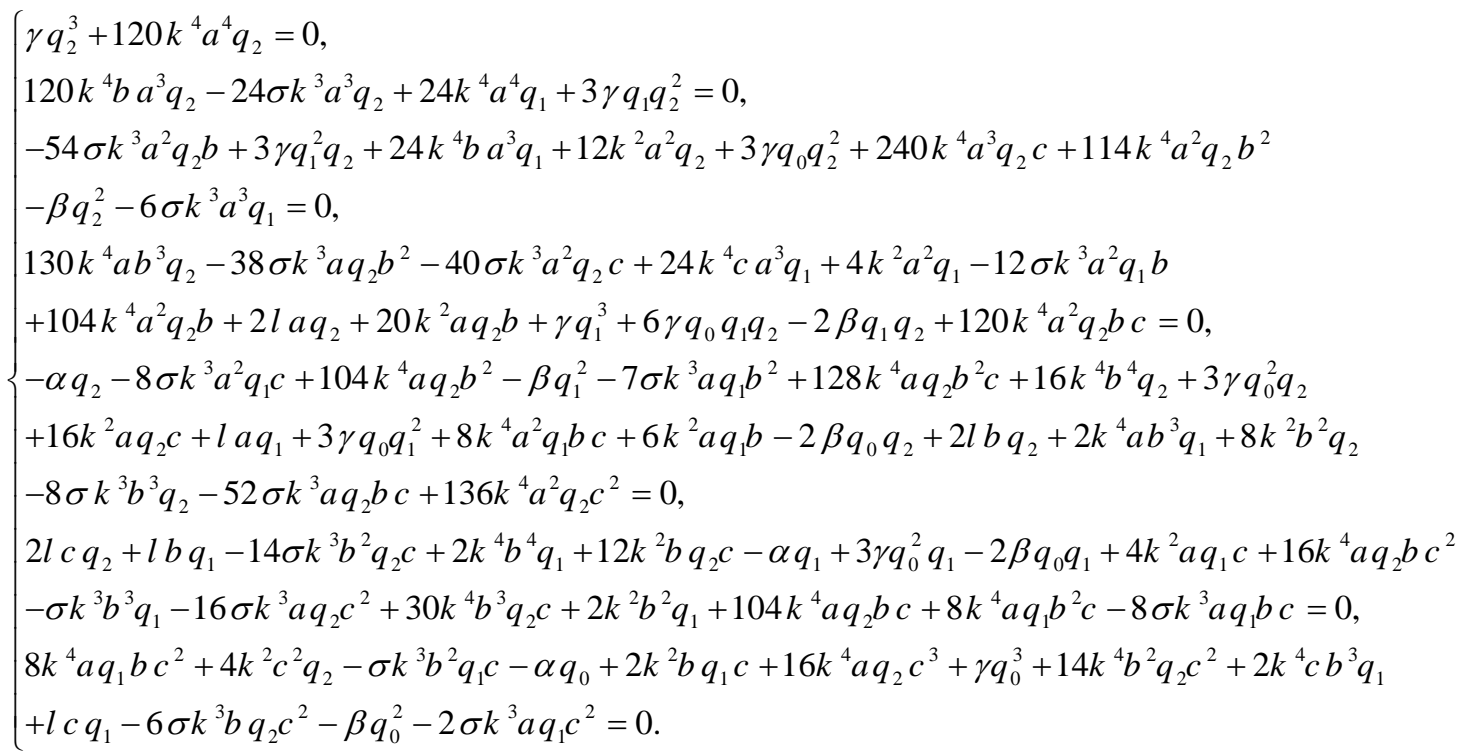

Solving this algebraic equation (By Maple 13), one finds

$$
\begin{aligned}
& q_{0}=\frac{1}{2940} \frac{-641 i \sigma k b \gamma \sqrt{30}+1252 i k^{2} b^{2} \gamma \sqrt{30}-23 i \gamma \sigma^{2} \sqrt{30}+196 i \gamma \sqrt{30} 3920 i k^{2} a c \gamma \sqrt{30}+980 \beta \sqrt{\gamma}}{\gamma^{\frac{3}{2}}}, \\
& q_{1}=\frac{\frac{1}{7} i k a \sqrt{30}(5 k b-\sigma)}{\sqrt{\gamma}}, \quad q_{2}=\frac{2 i \sqrt{30} k^{2} a^{2}}{\sqrt{\gamma}} .
\end{aligned}
$$

It is to be noted that the Riccati equation (5) can be solved using the homogeneous balance method as the following:

Tayp 1: Let $\phi=\sum_{i=0}^{m} b_{i} \tanh ^{i} \xi$. Balancing $\phi^{\prime}$ with $\phi^{2}$ leads to

$$
\phi=b_{0}+b_{1} \tanh \xi
$$

Substituing Eq. (8) into (5) results the following solution of Eq.(5)

$$
\phi=\frac{(-1)}{2 a}(b+2 \tanh \xi), \quad a c=\frac{b^{2}}{4}-1 .
$$

Substitution in (6), from Eqs. (7) and (9), leads to the following traveling wave solution of Eq. (1)

$$
\begin{aligned}
& u=\frac{1}{2940}\left(\frac{-641 i \sqrt{30} \gamma b \sigma k+1252 i k^{2} \sqrt{30} \gamma b^{2}-23 i \sigma^{2} \sqrt{30} \gamma+196 i \sqrt{30} \gamma+3920 i k^{2} a \sqrt{30} c \gamma+980 \beta \sqrt{\gamma}}{\gamma^{(3 / 2)}}\right. \\
& -\frac{\frac{1}{14} i k \sqrt{30}(-\sigma+5 k b)(b+2 \tanh \xi)}{\sqrt{\gamma}}+\frac{\frac{1}{2} i \sqrt{30} k^{2}(b+2 \tanh \xi)^{2}}{\sqrt{\gamma}},
\end{aligned}
$$


Similarly, if one takes $\phi=\sum_{i=0}^{m} b_{i} \operatorname{coth}^{i} \xi$, then we obtain the following traveling wave solutions of Eq.(1) will be obtain. $u=\frac{1}{2940}\left(\frac{-641 i \sqrt{30} \gamma b \sigma k+1252 i k^{2} \sqrt{30} \gamma b^{2}-23 i \sigma^{2} \sqrt{30} \gamma+196 i \sqrt{30} \gamma+3920 i k^{2} a \sqrt{30} c \gamma+980 \beta \sqrt{\gamma}}{\gamma^{(3 / 2)}}\right.$ $-\frac{\frac{1}{14} i k \sqrt{30}(-\sigma+5 k b)(b+2 \operatorname{coth} \xi)}{\sqrt{\gamma}}+\frac{\frac{1}{2} i \sqrt{30} k^{2}(b+2 \operatorname{coth} \xi)^{2}}{\sqrt{\gamma}}$,

Tayp 2: From [7], when $a=1$, the Riccati equation (5) has the following solutions

$$
\phi= \begin{cases}-\sqrt{-c} \tanh (\sqrt{-c} \xi), & c<0 \\ \frac{-1}{\xi}, & c=0 \\ \sqrt{c} \tan (\sqrt{c} \xi), & c>0\end{cases}
$$

From (6), (7), (12), one gets the following traveling wave solutions of Eq. (1).

When $c<0$,

$$
\begin{aligned}
& u=\frac{1}{2940} \frac{-641 i \sqrt{30} \gamma b \sigma k+1252 i k^{2} \sqrt{30} \gamma b^{2}-23 i \sigma^{2} \sqrt{30} \gamma+196 i \sqrt{30} \gamma+3920 i k^{2} a \sqrt{30} c \gamma+980 \beta \sqrt{\gamma}}{\gamma^{(3 / 2)}} \\
& -\frac{\frac{1}{7} i k a \sqrt{30}(-\sigma+5 k b) \sqrt{-c} \tanh (\sqrt{-c} \xi)}{\sqrt{\gamma}}-\frac{2 i \sqrt{30} a^{2} k^{2} \operatorname{ctanh}(\sqrt{-c} \xi)^{2}}{\sqrt{\gamma}}
\end{aligned}
$$

When $c=0$,

$$
\begin{aligned}
& u=\frac{1}{2940} \frac{-641 i \sqrt{30} \gamma b \sigma k+1252 i k^{2} \sqrt{30} \gamma b^{2}-23 i \sigma^{2} \sqrt{30} \gamma+196 i \sqrt{30} \gamma+3920 i k^{2} a \sqrt{30} c \gamma+980 \beta \sqrt{\gamma}}{\gamma^{(3 / 2)}} \\
& -\frac{\frac{1}{7} i k a \sqrt{30}(5 k b-\sigma)}{\sqrt{\gamma} \xi}+\frac{2 i \sqrt{30} k^{2} a^{2}}{\sqrt{\gamma} \xi^{2}}
\end{aligned}
$$

When $c>0$,

$$
\begin{aligned}
& u=\frac{1}{2940} \frac{-641 i \sqrt{30} \gamma b \sigma k+1252 i k^{2} \sqrt{30} \gamma b^{2}-23 i \sigma^{2} \sqrt{30} \gamma+196 i \sqrt{30} \gamma+3920 i k^{2} a \sqrt{30} c \gamma+980 \beta \sqrt{\gamma}}{\gamma^{(3 / 2)}} \\
& +\frac{\frac{1}{7} i k a \sqrt{30}(-\sigma+5 k b) \sqrt{c} \tanh (\sqrt{c} \xi)}{\sqrt{\gamma}}+\frac{2 i \sqrt{30} a^{2} k^{2} \operatorname{ctanh}(\sqrt{c} \xi)^{2}}{\sqrt{\gamma}}
\end{aligned}
$$

Tayp 3:lets find the solutions of the Riccati equation, (5), in the following form

$$
\phi=A_{0}+\sum_{i=1}^{m}\left(A_{i} f^{i}+B_{i} f^{i-1} g\right)
$$

Where $\quad f=\frac{1}{\cosh \xi+r}, \quad g=\frac{\sinh \xi}{\cosh \xi+r}$,

which satisfy

$$
\begin{aligned}
& f^{\prime}(\xi)=-f(\xi) g(\xi), \quad g^{\prime}(\xi)=1-g^{2}(\xi)-r f(\xi), \\
& g^{2}(\xi)=1-2 r f(\xi)+\left(r^{2}-1\right) f^{2}(\xi) .
\end{aligned}
$$

Balancing $\phi^{\prime}$ with $\phi^{2}$ leads to

$$
\phi=A_{0}+A_{1} f+B_{1} g .
$$

Substituting Eq. (17) into (5), collecting the coefficient of the terms with the same power, of $f^{i}(\xi) g^{j}(\xi)(i=0,1,2 ; j=0,1)$, and these coefficients, to zero yield to the following set of algebra equations 


$$
\left\{\begin{array}{l}
a A_{0}^{2}+a B_{1}^{2}+b A_{0}+c=0, \\
2 a A_{0} A_{1}-2 a r B_{1}^{2}-r B_{1}+b A_{1}=0, \\
a A_{1}^{2}+a\left(r^{2}-1\right) B_{1}^{2}+\left(r^{2}-1\right) B_{1}=0, \\
2 a A_{0} B_{1}+b B_{1}=0, \\
2 a A_{1} B_{1}+A_{1}=0 .
\end{array}\right.
$$

Which have solutions

$$
A_{0}=-\frac{b}{2 a}, \quad A_{1}= \pm \sqrt{\frac{\left(r^{2}-1\right)}{4 a^{2}}}, \quad B_{1}=\frac{-1}{2 a}, c=\frac{b^{2}-1}{4 a},
$$

From Eqs. (17) and (18), we have

$$
\phi=\frac{-1}{2 a}\left(b+\frac{\sinh \xi \mp \sqrt{\left(r^{2}-1\right)}}{\cosh \xi+r}\right)
$$

From Eqs. (6), (7) and (19), we obtain the new wave solutions of Eq. (1)

$u=\frac{1}{2940} \frac{-641 i \sqrt{30} \gamma b \sigma k+1252 i k^{2} \sqrt{30} \gamma b^{2}-23 i \sigma^{2} \sqrt{30} \gamma+196 i \sqrt{30} \gamma+3920 i k^{2} a \sqrt{30} c \gamma+980 \beta \sqrt{\gamma}}{\gamma^{(3 / 2)}}$

$-\frac{1}{14} \frac{k i \sqrt{30}(-\sigma+5 k b)\left(b+\frac{\left(\sinh \xi \mp \sqrt{r^{2}-1}\right.}{\cosh \xi+r}\right.}{\sqrt{\gamma}}+\frac{\frac{1}{2} i \sqrt{30} k^{2}\left(b+\frac{\sinh \xi \mp \sqrt{r^{2}-1}}{\cosh \xi+r}\right)^{2}}{\sqrt{\gamma}}$.

Tayp 4: We take $\phi$ in the Riccati equation (5) being of the form

$$
\phi=e^{p_{1} \xi} \rho(z)+p_{4}(\xi),
$$

Where $z=e^{p_{2} \zeta}+p_{3}$, where $p_{1}, p_{2}$, and $p_{3}$ are constants to be determined.

Substituting (21) into (5), we have

$$
\phi^{\prime}-a \phi^{2}-b \phi-c=p_{2} e^{\left(p_{1}+p_{2}\right) \xi} \rho^{\prime}-a e^{2 p_{1} \xi} \rho^{2}+\left(p_{1}-2 a p_{4}\right) e^{p_{1} \xi} \rho-a p_{4}^{2}-b e^{p_{1} \xi} \rho-b p_{4}-c=0
$$

Setting $p_{1}+p_{2}=2 p_{1}$, we get $p_{1}=p_{2}$. If we assume that $p_{4}=\frac{p_{1}-b}{2 a}$, and $c=\frac{-p_{1}^{2}+b^{2}}{4 a}$, then Eq. (22) becomes

$$
p_{2} \rho^{\prime}-a \rho^{2}=0
$$

Solving Eq.(23), we get

$$
\rho=\frac{-p_{1}}{a z}=-\frac{p_{1}}{a\left(e^{p_{1} \xi}+p_{3}\right)}
$$

Substituting (24) and $p_{4}=\frac{p_{1}-b}{2 a}$, into (21), we have

$$
\phi=-\frac{p_{1} e^{p_{1} \xi}}{a\left(e^{p_{1} \xi}+p_{3}\right)}+\frac{p_{1}-b}{2 a} .
$$

If $p_{3}=1$ in $(25)$, we have 


$$
\phi=-\frac{p_{1}}{2 a} \tanh \left(\frac{1}{2} p_{1} \xi\right)-\frac{b}{2 a} .
$$

If $p_{3}=-1$ in (25), we have

$$
\phi=-\frac{p_{1}}{2 a} \operatorname{coth}\left(\frac{1}{2} p_{1} \xi\right)-\frac{b}{2 a} .
$$

From (6), (7) and (25), we obtain the following new wave solutions of Eq. (1)

$$
\begin{aligned}
& u=\frac{1}{2940} \frac{-641 i \sqrt{30} \gamma b \sigma k+1252 i k^{2} \sqrt{30} \gamma b^{2}-23 i \sigma^{2} \sqrt{30} \gamma+196 i \sqrt{30} \gamma+3920 i k^{2} a \sqrt{30} c \gamma+980 \beta \sqrt{\gamma}}{\gamma^{(3 / 2)}} \\
& \frac{\frac{1}{7} i k a \sqrt{30}(-\sigma+5 k b)\left(-\frac{p_{1} e^{p_{1} \xi}}{a\left(e^{p_{1} \xi}+p_{3}\right)}+\frac{1}{2} \frac{p_{1}-b}{a}\right)}{\sqrt{\gamma}}+\frac{2 i k^{2} a^{2} \sqrt{30}\left(-\frac{p_{1} e^{p_{1} \xi}}{a\left(e^{p_{1} \xi}+p_{3}\right)}+\frac{1}{2} \frac{p_{1}-b}{a}\right)^{2}}{\sqrt{\gamma}}
\end{aligned}
$$

When $p_{3}=1$, we have from (26)

$u=\frac{1}{2940} \frac{-641 i \sqrt{30} \gamma b \sigma k+1252 i k^{2} \sqrt{30} \gamma b^{2}-23 i \sigma^{2} \sqrt{30} \gamma+196 i \sqrt{30} \gamma+3920 i k^{2} a \sqrt{30} c \gamma+980 \beta \sqrt{\gamma}}{\gamma^{(3 / 2)}}$

$\frac{\frac{1}{7} i k a \sqrt{30}(-\sigma+5 k b)\left(-\frac{1}{2} \frac{p_{1} \tanh \left(\frac{1}{2} p_{1} \xi\right)}{a}+\frac{1}{2} \frac{b}{a}\right)}{\sqrt{\gamma}}+\frac{2 i k^{2} a^{2} \sqrt{30}\left(-\frac{1}{2} \frac{p_{1} \tanh \left(\frac{1}{2} p_{1} \xi\right)}{a}+\frac{1}{2} \frac{b}{a}\right)^{2}}{\sqrt{\gamma}}$

When $p_{3}=-1$, we have from (27)

$$
\begin{aligned}
& u=\frac{1}{2940} \frac{-641 i \sqrt{30} \gamma b \sigma k+1252 i k^{2} \sqrt{30} \gamma b^{2}-23 i \sigma^{2} \sqrt{30} \gamma+196 i \sqrt{30} \gamma+3920 i k^{2} a \sqrt{30} c \gamma+980 \beta \sqrt{\gamma}}{\gamma^{(3 / 2)}} \\
& \frac{\frac{1}{7} i k a \sqrt{30}(-\sigma+5 k b)\left(-\frac{1}{2} \frac{p_{1} \operatorname{coth}\left(\frac{1}{2} p_{1} \xi\right)}{a}+\frac{1}{2} \frac{b}{a}\right)}{\sqrt{\gamma}}+\frac{2 i k^{2} a^{2} \sqrt{30}\left(-\frac{1}{2} \frac{p_{1} \operatorname{coth}\left(\frac{1}{2} p_{1} \xi\right)}{a}+\frac{1}{2} \frac{b}{a}\right)^{2}}{\sqrt{\gamma}}
\end{aligned}
$$

Clearly, (10), (11) is the special case of (29), (30) with $p_{1}=2$.

Tayp 5: We suppose that the Riccati equation (5) have the following solutions of the form

$$
\phi=A_{0}+\sum_{i=1}^{m} \sinh ^{i-1}\left(A_{i} \sinh \omega+B_{i} \cosh \omega\right)
$$

Where $\frac{d \omega}{d \xi}=\sinh \omega$ or $\frac{d \omega}{d \xi}=\cosh \omega$. Balancing $\phi^{\prime}$ with $\phi^{2}$ leads to

$\phi=A_{0}+A_{1} \sinh \omega+B_{1} \cosh \omega$

When $\frac{d \omega}{d \xi}=\sinh \omega$, lets substitue (31) into (5) and set the coefficient of $\sinh ^{i} \omega \cosh ^{i} \omega(i=0,1,2 ; j=0,1)$ to zero. A set of algebraic equations is obtained as follows

$$
\left\{\begin{array}{l}
a A_{0}^{2}+a B_{1}^{2}+b A_{0}+c=0, \\
2 a A_{0} A_{1}+b A_{1}=0, \\
a A_{1}^{2}+a B_{1}^{2}=B_{1}, \\
2 a A_{0} B_{1}+b B_{1}=0, \\
2 a A_{1} B_{1}+A_{1}=0,
\end{array}\right.
$$

for which, we have the following solutions 


$$
A_{0}=\frac{-b}{2 a}, \quad A_{1}=0, \quad B_{1}=\frac{1}{a},
$$

Where $c=\frac{b^{2}-4}{4 a}$, and

$$
A_{0}=\frac{-b}{2 a}, A_{1}= \pm \sqrt{\frac{1}{2 a}}, B_{1}=\frac{1}{2 a},
$$

Where $c=\frac{b^{2}-1}{4 a}$.

By the hypothesis $\frac{d \omega}{d \xi}=\sinh \omega$, one gets

$$
\sinh \omega=-\operatorname{csch} \xi, \quad \cosh \omega=-\operatorname{coth} \xi .
$$

From (31), (33), (35), and $c=\frac{b^{2}-4}{4 a}$, one reads

$$
\phi=-\frac{b+2 \operatorname{coth} \xi}{2 a}
$$

From (31), (34), (35), and $c=\frac{b^{2}-1}{4 a}$

$$
\phi=-\frac{b \pm \csc h \xi+\operatorname{coth} \xi}{2 a},
$$

From (5), (6), (7), (36) and (37), a new traveling wave solutions of Eq. (1), will be resulted

$$
\begin{aligned}
& u=\frac{1}{2940} \frac{-641 i \sqrt{30} \gamma b \sigma k+1252 i k^{2} \sqrt{30} \gamma b^{2}-23 i \sigma^{2} \sqrt{30} \gamma+196 i \sqrt{30} \gamma+3920 i k^{2} a \sqrt{30} c \gamma+980 \beta \sqrt{\gamma}}{\gamma^{(3 / 2)}} \\
& -\frac{\frac{1}{14} i k \sqrt{30}(-\sigma+5 k b)(b+2 \operatorname{coth} \xi)}{\sqrt{\gamma}}+\frac{\frac{1}{2} i \sqrt{30} k^{2}(b+2 \operatorname{coth} \xi)^{2}}{\sqrt{\gamma}},
\end{aligned}
$$

Where $c=\frac{b^{2}-4}{4 a}$, and

$$
\begin{aligned}
& u=\frac{1}{2940} \frac{-641 i \sqrt{30} \gamma b \sigma k+1252 i k^{2} \sqrt{30} \gamma b^{2}-23 i \sigma^{2} \sqrt{30} \gamma+196 i \sqrt{30} \gamma+3920 i k^{2} a \sqrt{30} c \gamma+980 \beta \sqrt{\gamma}}{\gamma^{(3 / 2)}} \\
& -\frac{\frac{1}{14} i k \sqrt{30}(-\sigma+5 k b)(b-\csc h \xi+\operatorname{coth} \xi)}{\sqrt{\gamma}}+\frac{\frac{1}{2} i \sqrt{30} k^{2}(b-\csc h \xi+\operatorname{coth} \xi)^{2}}{\sqrt{\gamma}},
\end{aligned}
$$

Where $c=\frac{b^{2}-1}{4 a}$.

Similary, when $\frac{d \omega}{d \xi}=\cosh \omega$, 


$$
\begin{aligned}
& u=\frac{1}{2940} \frac{-641 i \sqrt{30} \gamma b \sigma k+1252 i k^{2} \sqrt{30} \gamma b^{2}-23 i \sigma^{2} \sqrt{30} \gamma+196 i \sqrt{30} \gamma+3920 i k^{2} a \sqrt{30} c \gamma+980 \beta \sqrt{\gamma}}{\gamma^{(3 / 2)}} \\
& -\frac{\frac{1}{14} i k \sqrt{30}(-\sigma+5 k b)(b+2 \cot \xi)}{\sqrt{\gamma}}+\frac{\frac{1}{2} i \sqrt{30} k^{2}(b+2 \cot \xi)^{2}}{\sqrt{\gamma}},
\end{aligned}
$$

Where $c=\frac{b^{2}-4}{4 a}$, and

$$
\begin{aligned}
& u=\frac{1}{2940} \frac{-641 i \sqrt{30} \gamma b \sigma k+1252 i k^{2} \sqrt{30} \gamma b^{2}-23 i \sigma^{2} \sqrt{30} \gamma+196 i \sqrt{30} \gamma+3920 i k^{2} a \sqrt{30} c \gamma+980 \beta \sqrt{\gamma}}{\gamma^{(3 / 2)}} \\
& -\frac{\frac{1}{14} i k \sqrt{30}(-\sigma+5 k b)(b-\csc \xi+\cot \xi)}{\sqrt{\gamma}}+\frac{\frac{1}{2} i \sqrt{30} k^{2}(b-\csc \xi+\cot \xi)^{2}}{\sqrt{\gamma}},
\end{aligned}
$$

Where $c=\frac{b^{2}-1}{4 a}$.

\section{Conclusion}

Lets summarize the HBM has been used, to solve the Swift-Hohenberg equation, exact traveling solution of the equation are resulted.

Step 1: For a given nonlinear evolution equation

$$
F\left(u, u_{t}, u_{x}, u_{t t}, u_{x t}, u_{x x}, u_{x x t}, u_{x t t}, u_{x x x}, u_{t t t}\right)=0 .
$$

we consider the transformation $u(x, t)=u(\xi), \xi=k x+l t+d$, to reduce Eq. (40) into an nonlinear ordinary differential equation

$$
Q\left(u, l u^{\prime}, k u^{\prime}, l^{2} u^{\prime \prime}, k l u^{\prime \prime}, k^{2} u^{\prime \prime}, k^{2} l u^{\prime \prime \prime}, k l^{2} u^{\prime \prime \prime}, k^{3} u^{\prime \prime \prime}, l^{3} u^{\prime \prime \prime}\right)=0 .
$$

Step 2: For a given ansatz equation (for example, the ansatz equation is $\phi^{\prime}=a \phi^{2}+b \phi+c$ in this paper), the form of $u$ is considered as a truncated power series, in powers of $\phi$ and the homogeneous balance method is used on Eq. (41) to find the coefficients of this.series

Step 3: The homogeneous balance method is used to solve the ansatz equation.

Step 4: Finally, the traveling wave solutions of Eq. (40) are obtained by combining step 2 and 3.

From the above procedure, it is easy to find that the homogeneous balance method is more effective and simple than other methods and many solutions can be obtained at the same time. This method can be also applied to other nonlinear evolution equations. 


\section{References}

[1] Wang ML,solitary wave solutions for variant Boussinesq equations, Phys. Lett. A (1995), Vol.199, pp.169-172.

[2] Wang ML,Exact solutions for a compound Kdv-Burgers equation, Phys Lett A (1996), vol.213, pp.279-287.

[3] Wang ML, Zhou YB, Li ZB. Application a homogeneous balance method to exact solutions of nonlinear equations in mathematical physics, Phys Lett A (1996), vol.216. pp.67-75.

[4] E.G. Fan, H.Q. Zhang, A note on the homogeneous balance method, Phys. Lett. A (1998), vol.246, pp.403-406.

[5] Liu CQ, Zhou RG, Zhou MR. Phys Lett 1998; 246:113.

[6] Zhao XQ, Tang DB, Wang LM, Zhang YM. Acta Phys Sin 2003;52: 1827.

[7] X.Q. Zhao, D.B. Tang, A new note on a homogeneous balance method, Phys. Lett. A (2002), vol.297, pp. 59-67. 